\title{
Formation du plasma dans une décharge capillaire rapide assistée par effet de cathode creuse
}

\author{
I. Krisch ${ }^{1,2}$, P. Choi ${ }^{1}$, J. Larour ${ }^{1}$, J. Rous ${ }^{1}$, C. Dumitrescu ${ }^{1,3}$, A. Chuvatin ${ }^{1}$, \\ S. Zakharov ${ }^{3}$, C. Leblanc ${ }^{3}$ et M. Favre ${ }^{4}$ \\ ${ }^{1}$ Laboratoire de Physique des Milieux lonisés, UMR 7648 du CNRS, École Polytechnique, \\ 91128 Palaiseau, France \\ 2 John Caunt Scientific Ltd., Oxford OX9 2QA, U.K. \\ ${ }^{3}$ EPPRA, XTEC, École Polytechnique, 91128 Palaiseau, France \\ ${ }^{4}$ Facultad de Fisica, Pontificia Universidad Catolica de Chile, Santiago 22, Chile
}

\begin{abstract}
Résumé : La combinaison des caractères d'une décharge capillaire rapide avec ceux d'une décharge transitoire à cathode creuse a permis d'étudier une famille nouvelle de micro-plasmas. De telles décharges fonctionnent différemment des décharges capillaires usuelles décrites dans la littérature. Un faisceau d'électrons résultant de l'effet de cathode creuse transitoire crée une préionisation sur l'axe, favorable à la formation de la décharge. La paroi du capillaire agit alors seulement comme un limiteur et le matériau de la paroi ne contamine pas le plasma, sauf aux temps longs, dans la mesure où le temps de montée initial du courant est suffisamment élevé. Une géométrie particulière des électrode assure la très faible inductance indispensable pour réaliser cette condition. Un courant de décharge typique de plusieurs kA alimente la décharge avec un temps de montée nanoseconde, produisant une colonne de plasma chaud de taille sub-millimétrique qui rayonne de la région UVV aux X mous. Des mesures complètes de spectroscopie UVV ont été faites pour caractériser le plasma. L'évolution temporelle du diamètre de la source est mesurée par une technique de caméra fente-fil, et montre que le processus trouve bien son origine sur l'axe.
\end{abstract}

Les décharges capillaires rapides (DCR) sont maintenant connues pour être des émetteurs puissants dans le domaine UVX. Cependant une catégorie, les DCR non ablatives, reste peu étudiée bien qu'elle présente des performances remarquables pour l'efficacité de conversion de l'énergie électrique en photonique. Le dispositif étudié par les partenaires a été décrits plusieurs fois, en particulier quant au processus de formation du plasma [1-3]. Dans le travail présenté ici, on cherche à montrer que l'utilisation de l'effet de cathode creuse est un moyen unique d'initier une décharge sur l'axe et de confiner au mieux le plasma pour le chauffer en un temps très court.

Le dispositif expérimental [3] est basé sur la décharge pulsée d'un banc de $6 \mathrm{nF}$ sous $18 \mathrm{kV}$ dans une cellule parfaitement coaxiale formant condensateur. Le stockage transitoire de l'énergie est fait aux extrémités du capillaire grâce à une géométrie où les armatures de la cellule forment un condensateur de capacité propre entre 1 et $4 \mathrm{nF}$ environ. Une arrivée de gaz ( $\mathrm{Ar}, \mathrm{Xe}, \mathrm{CH} 4$ sous une fraction de mTorr) par la cathode et un pompage secondaire par l'anode ainsi qu'une structure original de la cathode assurent une forte dissymétrie de la cellule de décharge. Deux types de capillaire en alumine on été utilisés, avec des diamètres intérieurs de 0,8 et $1,6 \mathrm{~mm}$ respectivement, pour une longueur de $12 \mathrm{~mm}$. La cellule peut présenter un auto-déclenchement mais on préfêre le contrôler par l'adjonction dans l'espace cathodique arrière, près de l'extrémité du capillaire, d'un canon à plasma alimenté avec un léger retard par limpulsion de tension de charge. On mesure le courant par une gorge inductive [3], le tension par un diviseur résistif et on place sur l'axe dans le vide une sonde polarisée négativement qui peut montrer un effet photoélectrique, collecter les ions positifs mais aussi collecter les électrons qui auraient une forte énergie. L'examen de traces typiques pour plusieurs pressions d'Ar (Figure 1) montre bien le claquage de la cellule et la très rapide montée de courant. Mais il faut noter sur la réponse de la sonde un signal d'électrons entrants, de signe opposé au grand pic photoélectrique, signe d'un faisceau d'électrons énergétiques [1-3]. Un examen systématique montre qu'un fonctionnement efficace de la décharge pour la conversion XUV nécessite, en mode déclenché, la formation d'un faisceau d'électrons énergétiques (de l'ordre de la tension de polarisation) qui préionise le gaz et assure les conditions de la croissance rapide du courant. On peut noter aussi qu'il existe un optimum de pression pour ce type de décharge, principalement lié à la difficulté de créer le chauffage par faisceau d'électrons à trop basse pression (pas assez d'intensité) ou à trop haute pression (trop d'atomes cibles. On observe alors spectroscopiquement des ions ArX ou XeX.

Une mesure complémentaire a été faite par la méthode de la caméra fente-fil [4] qui, par un système d'imagerie par des fentes bloquées par des fils de diamètres variés, permet de donner la taille de la source par analyse des pénombres. Une capture d'image par galette de microcanaux pulsés et une filtrage 
énergétique large bande par des absorbeurs permettent de voir l'évolution du rayon. La Figure 2 montre que le canal chaud part bien de l'axe, et croît d'autant plus lentement que le filtrage est dur, c'est à dire que le plasma est chaud.

La conclusion de cette étude la formation du plasma, initié par faisceau d'électron dû à l'effet de cathode creuse, est que le procédé étudié est un moyen unique de réaliser un plasma sans pollution par l'ablation des parois. Ceci est favorable à la pureté des spectres observés et à la haute température du plasma observée ( 30 à $60 \mathrm{eV}$ ) par la faiblesse du nombre d'atomes à ioniser. Les études actuelles portent sur les spectres émis, en particulier dans le domaine UV extrême, sur l'augmentation de l'énergie stockée et sur le taux de répétition, actuellement de l'ordre du $\mathrm{Hz}$.
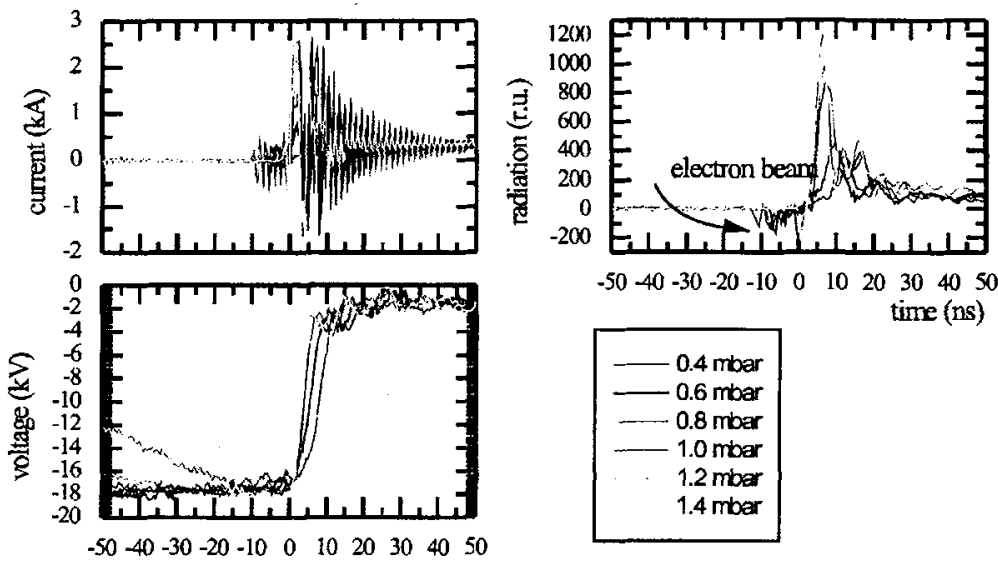

Figure 1 : Signaux électriques typiques de courant (kA), tension (kV) et de la sonde photoélectique (u.a.) pour une décharge dans l'argon, pour plusieurs pressions cathodiques.



Figure 2 : Dépendance temporelle du rayon de la décharge dans l'Argon pour un capillaire de rayon 0,4mm.

\section{Remerciements}

Ce travail est soutenu par la commission Européenne, contrat TMR 980186 (FACADIX) et un accord CNRS-CONICYT. La construction mécanique doit beaucoup à B. Dufour.

\section{Références}

1. P. Choi, H. Chuaqui, M. Favre, V. Colas, IEEE Trans. Plasma Sci. 23, 221 (1995)

2. M. Favre, P. Choi, H. Chuaqui, Y. Kaufman, J. Moreno, E. Wyndham, M. Zambra, IEEE Trans. Plasma Sci. 23, 212 (1995)

3. P. Choi and M. Favre, Review Scientific Instr. 69, 3118 (1998)

4. P.Choi, C. Dumitrescu-Zoita, B. Etlicher, A.V.Shislov, Proc. Xth IEEE Int. Pulsed Power Conference, Albuquerque, NM USA , Baker et Cooperstein editeurs p.886 (1995) 